\title{
Mitochondrial Fatty Acid Transport System and its Relevance to Ovarian Function
}

\author{
Mona Sharma \\ Department of Reproductive Biology, All India Institute of Medical Sciences, New Delhi-110029, India
}

Received: 06/12/2017_ Accepted: 1/03/2018_ Published: 20/03/2018

\begin{abstract}
Mitochondrial fatty acid oxidation is integral to folliculogenesis and oocyte maturation. Mitochondrial fatty acid transport system includes molecules such as 3-hydroxy-4-trimethylamino butyrate or carnitine, organic cation transporter novel 2 (OCTN2), carnitine palmitoyl transferase (CPT1), CPT2, and carnitine-acylcarnitine translocase (CACT). These are essential molecules that play an important role in the transport of activated long chain fatty acids from cytosol to the mitochondrial matrix where these subsequently undergo beta-oxidation. It has been well established that fatty acid metabolism is vital for follicles and oocyte growth. This review highlights the possible role of mitochondrial fatty transport acid system in ovarian function.
\end{abstract}

Keywords: Oocytes, Carnitine, Mitochondria, Fatty acids

\section{Introduction}

Mitochondrial function is essential for various cellular activities including cell division and cell death or apoptosis (1). Mitochondrial role has also been proven in human fertility (2). Fertility is a key component of reproductive health in men and women. Infertility has become a global health issue. Prevalence of infertility in female is estimated to be $15 \%$ in India (3). Ovarian function is integral to female fertility. Ovarian folliculogenesis needs energy from various sources like glucose, lipids and amino acids. Maximum energy is derived from mitochondrial fatty acids oxidation (4). Role of mitochondrial fatty acid $\beta$-oxidation is important in acquisition of oocyte developmental competence.

\section{Mitochondrial Fatty Acid Transport System}

Mitochondrial fatty acid transport system includes molecules such as 3-hydroxy-4-trimethylamino butyrate or carnitine, organic cation transporter novel 2 (OCTN2), carnitine palmitoyl transferase (CPT1), CPT2, and carnitineacylcarnitine translocase (CACT). The molecules play an important role in the transport of activated long chain fatty acids from cytosol to the mitochondrial matrix where these subsequently undergo beta-oxidation. Fatty acid transporter or carnitine plays an essential role in the transport of activated fatty acids across the inner mitochondrial membrane (5). Carnitine is 3-hydroxy-4-trimethylaminobutyrate and exists in $\mathrm{L}$ and $\mathrm{D}$ isomers. Normal serum L-carnitine levels are between $25-50 \mu \mathrm{M}$. Carnitine is synthesized from lysine and methionine mainly in kidney, liver and brain (6). Free carnitine was first isolated from bovine muscle and only L- isomer was found to be bioactive. L-carnitine is also involved in maintaining balance by removing accumulated toxic fatty acyl-CoA metabolites. Carnitine is reabsorbed in circulation by renal tubular active transport by OCTN2 transporter protein regulated by SLC22A5 gene. OCTN2 is essential for carnitine homeostasis. The fatty acids are first conjugated to form acyl CoA. This acyl group is then transferred to carnitine forming acyl-carnitine by CPTI present in inner mitochondrial membrane (figure 1). Acyl-carnitine is transferred to matrix via CACT. After traversing membrane, acyl group is transferred by CPT2 from carnitine to CoA which then undergoes citric acid cycle. Carnitine is then shuttled back across membrane by CACT and the process is repeated (7). TMLHE (epsilontrimethyllysine hydroxylase) is the gene encoding the first enzyme-trimethyl lysine dioxygenase/hydroxylase, catalyzing the rate limiting step of carnitine biosynthesis. Trimethyllysine dioxygenase /hydroxylase is a non-heme ferrous iron hydroxylase and 2-oxoglutarate dependent. It is the only enzyme of carnitine biosynthesis located in the mitochondria whereas the other three are cytosolic. The encoded protein converts trimethyllysine (TML) into hydroxytrimethyllysine (HTML) in mitochondria. Further, trimethylaminobutaraldehyde dehydrogenase converts HTML into trimethylamino- butaraldehyde (TAB). Hydroxytrimethyllysine aldolase converts TAB into trimethylaminobutyrate/butyrobetaine which is catalyzed by $\mathrm{Y}$ butyrobetaine dioxygenase and finally converted into carnitine (7).

\section{Relevance to Ovarian Function}

Successful reproduction depends on competent oocyte capable of undergoing fertilization. Role of carnitine through fatty acid metabolism has also been established in oocyte growth and development. The antiapoptotic property of l-carnitine is to stabilize mitochondrial membrane thereby increasing supply of energy. Oocyte meiotic maturation needs energy from various sources like glucose, lipids and amino acids. Oocyte maturation includes cytoplasmic and nuclear events all of which are energy consuming and require adequate ATP (8). Oxidative stress is one of the mediators that affect quantity and quality of oocytes by inducing apoptosis. Antioxidant supplementation has been proved to protect oocytes against reactive oxygen species (ROS) and oxidative stress.

Corresponding author: Mona Sharma, Assistant Professor, Department of Reproductive Biology, All India Institute of Medical Sciences, New Delhi-110029, India. Contact No. 9968147821. Email address:dr.mona18sharma@gmail.com 


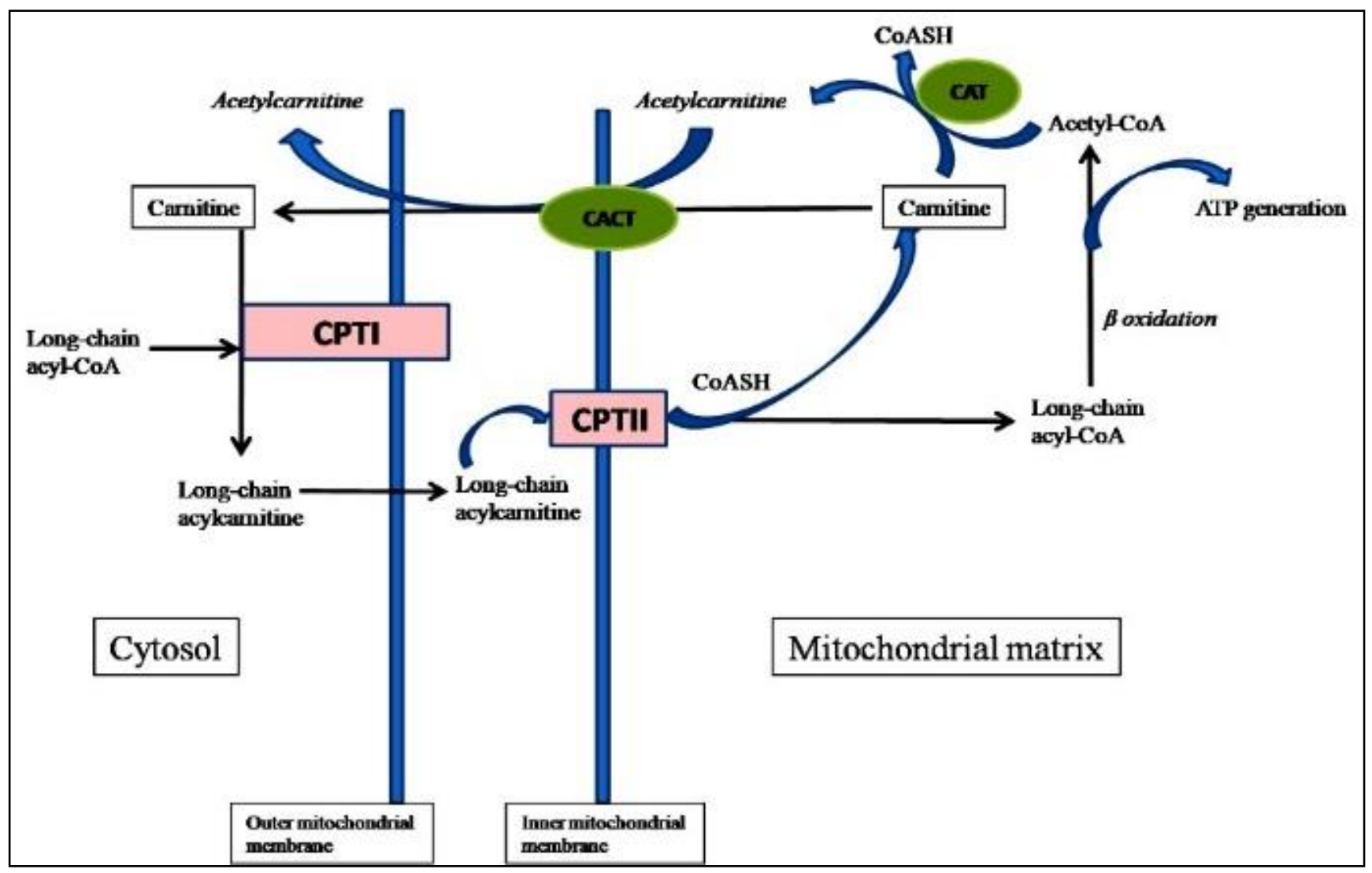

Figure 1. Schematic diagram showing carnitine mediated transfer of fatty acids across mitochondrial membrane

Carnitine is also an antioxidant that protects mitochondria from oxidative stress. The role of carnitine has been well proved in oocyte maturation. Fatty acid oxidation is critical for oocyte maturation as shown in mice (9). Inhibition of fatty acid oxidation with CPTI inhibitor etomoxir during in-vitro maturation delays oocyte meiotic maturation (10). Moreover, carnitine supplementation of culture media increases fatty acid oxidation that significantly improves oocyte quality in terms of fertilization and embryo development in mice and cows $(11,12)$. Fatty acid metabolism in cumulus cells has also been shown importance. There is enhanced expression of genes in cumulus cells related to fatty acid metabolism in mature oocytes as compared to immature oocytes (13). Increase in free fatty acid levels leads to mitochondrial dysfunction leading to cell death, oxidative stress and ROS generation. These effects are improved by carnitine treatment (14). Commonly used antioxidants are acetyl-Lcarnitine (ALCAR), sodium citrate and $\alpha$-lipoic acid $(15,16)$. ALCAR treatment of oocytes during in-vitro maturation (IVM) improves oocyte quality by increasing number of mature oocytes. This also improves normal spindle formation (17). Effects of fatty acid oxidation on nuclear maturation have also been shown in mice, cows and pigs (10). Variable ATP levels affect oocyte quality, embryo development and subsequent implantation (18). Mouse oocytes with inadequate ATP levels have also shown chromatin and microtubular abnormalities (19). Mitochondrion is also a signal transducer of apoptosis pathway. L-carnitine treatment has shown to inhibit apoptosis both in-vivo and in-vitro $(20,21)$. $\beta$-oxidation is important in acquisition of oocyte developmental competence and female fertility. L-carnitine supplementation has improved oocyte cytoskeleton damage and mitochondrial disruption (22). Beta-oxidation is required for oocyte meiotic resumption and nuclear maturation in mice and porcine oocytes (10).

\section{Clinical Implications and Future Prospects}

The association of abnormal functioning of fatty acid system has been observed in number of studies. CPT1A inhibition leads to decreased beta-oxidation and low number of blastocyst formation following oocyte fertilization (9). CPT2 gene mutation was associated with infertility (23). Not much work has been done on SLC22A5 gene coding for OCTN2 regulation. Role of CACT in reproduction is also not much elaborated. Few studies using microarray identified copy number variations in the TMLHE locus Xq28 in patients of premature ovarian failure (POF) (24).

Fertility potential of a woman with POF is mostly compromised by the time when the clinical manifestations of hormonal decline are yet to appear (25). POF is a primary ovarian defect with symptoms of absent menarche or cessation of/irregular menstruation, infertility and symptoms of physiological menopause due to low estrogen levels before the age of 40 years. Infertility in POF is caused by decreased oocyte reserve due to accelerated follicular and oocyte atresia or decreased ovarian reserve since birth. POF constitutes roughly $1 \%$ cases of infertility in females (26). Despite of multiple etiologies suggested such as genetic, metabolic, infections, autoimmune etc., POF remains idiopathic and untreated in most cases (27).

By looking at the function of mitochondrial fatty acid system in beta-oxidation and role of fatty acid oxidation in follicular and oocyte development, association of mitochondrial fatty acid system in pathophysiology of POF can be suggested. The routine tests fail to detect POF at earlier stages where chances of fertility improvement may persist 
Moreover, treatment options are lacking in POF. Therefore, search on early diagnostic markers and treatment options is utmost important. The future research should focus creating POF disease models using factors regulating mitochondrial fatty acid transport system. Identification of novel gene mutations in SLC22A5, TMLHE, CPT1A, CPT2, CACT will help in structuring biomarker gene panel for early identification of idiopathic POF. Validation studies in larger number of cohorts can mark the gene panel as biomarker of metabolic etiology of POF. This will help in identifying POF at an earlier stage where fertility preservation is possible. Designing supplement options based on regulating molecules of fatty acid transport system can also be suggested. But the disease models must be validated further using the biochemical and genetic inhibitors of molecules of fatty acid transport system.

\section{Conclusion}

Fatty acid metabolism being the most important energy source for folliculogenesis raises the possibility of its association in ovarian disorders such as POF. Fatty acid transport system in mitochondria is integral for efficient beta oxidation. Therefore, studying their association in ovarian functions will be a potential source of scientific information in future.

\section{Ethical issue}

Authors are aware of, and comply with, best practice in publication ethics specifically with regard to authorship (avoidance of guest authorship), dual submission, and manipulation of figures, competing interests and compliance with policies on research ethics. Authors adhere to publication requirements that submitted work is original and has not been published elsewhere in any language.

\section{Competing interests}

The authors declare that there is no conflict of interest that would prejudice the impartiality of this scientific work.

\section{Authors' contribution}

All authors of this study have a complete contribution for data collection, data analyses and manuscript writing.

\section{References}

1. Sharma M, Kumar R, Dhingra R. Apoptosis: Understanding of the signaling pathways. International Journal of Biological and Medical Research. 2012;3(3):2029-2031

2. Barbhuiya PN, Gogoi A, Ahmed G3, Mahanta R. Prevalence of Mitochondrial DNA Nucleotide Substitution Mutations in Male Infertile Cases of Northeast India. Journal of Infertility and Reproductive Biology. 2016;(4)1:11-21.

3. Rafique, S, Sterling EW, Nelson LM. A New Approach to Primary Ovarian Insufficiency. Obstetetrics and Gynecology Clinics of North America. 2012;39 (4):567-586.

4. Sturmey RG, Reis A, Leese HJ, McEvoy TG. Role of fatty acids in energy provision during oocyte maturation and early embryo development. Reproduction in Domestic Animals. 2009;44(Suppl 3):50-58.

5. Monfregola J, Cevenini A, Terracciano A, et al. Functional analysis of TMLH variants and definition of domains required for catalytic activity and mitochondrial targeting. Journal of Cell Physiology. 2005;204:839-847.

6. Sharma S, Black SM. Carnitine homeostasis, mitochondrial function, and cardiovascular disease. Drug Discovery Today. Disease
Mechanisms. 2009; 6(1-4): e31-e39.

7. Vaz FM, Wanders RJA. Carnitine biosynthesis in mammals. The Biochemical Journal. 2002;361(Pt 3):417-429.

8. Mermillod P, Oussaid B, Cognie Y. Aspects of follicular and oocyte maturation that affect the developmental potential of embryos. Journal of Reproduction and Fertility. 1999;54:449-460.

9. Dunning KR, Cashman K, Russell DL, et al. b-oxidation is essential for mouse oocyte developmental competence and early embryo development. Biology of Reproduction. 2010;83:909-918.

10. Paczkowski M, Silva E, Schoolcraft WB, Krisher RL. Comparative importance of fatty acid b-oxidation to nuclear maturation, gene expression, and glucose metabolism in mouse, bovine, and porcine cumulus-oocyte complexes. Biology of Reproduction. 2013; 88(5):111.

11. Dunning KR, Akison LK, Russell DL, et al. Increased beta-oxidation and improved oocyte developmental competence in response to 1carnitine during ovarian in vitro follicle development in mice. Biology of Reproduction. 2011;85(3):548-555.

12. Sutton-McDowall ML, Feil D, Robker RL, et al. Utilization of endogenous fatty acid stores for energy production in bovine preimplantation embryos. Theriogenology. 2012;77:1632-1641.

13. Charlier C, Montfort J, Chabrol O, et al. Oocyte-somatic cells interactions, lessons from evolution. BMC Genomics. 2012;13:560.

14. ChangB, Nishikawa M, Nishiguchi S, et al. L-carnitine inhibits hepatocarcinogenesis via protection of mitochondria. International Journal of Cancer. 2005;113(5):719-729.

15. Gulcin I. Antioxidant and antiradical activities of L-carnitine. Life Sciences. 2006;78:803-811

16. Truong TT, Soh YM, Gardner DK. Antioxidants improve mouse preimplantation embryo development and viability. Human Reproduction. 2016;31:1445-1454.

17. Liang LF, Qi ST, Xian YX, et al. Protective effect of antioxidants on the prematuration aging of mouse oocytes. Scientific reports. 2017; $7: 1434$.

18. Van Blerkom J, Davis PW, Lee J. ATP content of human oocytes and developmental potential and outcome after in vitro fertilization and embryo transfer. Human Reproduction. 1995;10(2):415-424

19. Johnson MT, Freeman EA, Gardner DK, et al Oxidative metabolism of pyruvate is required for meiotic maturation of murine oocytes in vivo. Biology of Reproduction. 2007;77(1):2-8.

20. Chang B, Nishikawa M, Sato E, et al. L-Carnitine inhibits cisplatininduced injury of the kidney and small intestine. Archives of Biochemistry and Biophysics. 2002;405(1):55-64.

21. Pillich RT, Scarcella G, Risuleo G. Reduction of apoptosis through the mitochondrial pathway by the administration of acetyl-Lcarnitine to mouse fibroblasts in culture. Experimental Cell Research. 2005;306(1):1-8

22. Mansour G, Abdelrazeik H, Sharma RK, et al. L-carnitine supplementation reducesoocyte cytoskeleton damage andembryoapoptosisinduced by incubation in peritoneal fluid from patients with endometriosis. Fertility and sterility. 2009; 91(5 Suppl):2079-2086.

23. Hull ML, Nemeth D, Hague WM, et al. Mitochondrial fatty acid transport enzyme deficiency - implications for in vitro fertilization. Fertility and Sterility.2009;91:2079-2086

24. Aboura A, Dupas C, Tachdijan G, et al. Array comparative genomic hybridization profiling analysis reveals deoxyribonucleic acid copy number variations associated with premature ovarian failure. Journa of Clinical Endocrinology and Metabolism. 2009; 94(11):4540-4546.

25. Shelling AN. Premature ovarian failure. Reproduction. 2010:140(5):633-641.

26. Cordts E, Christofolini D, Amaro dos Santos A, et al. Genetic aspects of premature ovarian failure: a literature review. Archives of Gynecology and Obstetrics. 2011;283:635-643.

27. Celestino-Soper PBS, Shaw CA, Sanders SJ, et al. Use of array CGH to detect exonic copy number variants throughout the genome in autism families detects a novel deletion in TMLHE. Human Molecular Genetics. 2011;20:4360-4370. 